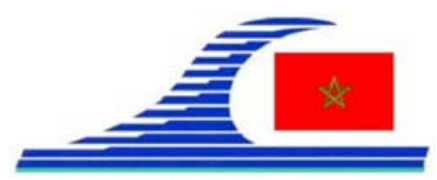

Conférence Méditerranéenne Côtière et Maritime EDITION 2, TANGER, MAROC (2011)

Coastal and Maritime Mediterranean Conference

Disponible en ligne - http://www.paralia.fr - Available online

\title{
Répartition de deux espèces introduites à caractère invasif dans la région centre de la côte algéroise : Caulerpa racemosa et Oculina patagonica
}

\author{
Souad LAMOUTI ${ }^{1}$, Chafika REBZANI ${ }^{2}$, Nour El Islam BACHARI ${ }^{2}$
}

1. Centre National de recherche de pêche et aquaculture Bous-smail Alger, Algérie. souad.lamouti@gmail.com

2. Faculté des Sciences Biologiques; Université des Sciences et Technologie Houari Boumedienne, ElAlia Bab Ezzouar Alger, Algérie.

bachari10@yahoo.fr,rebzanichafika@yahoo.fr

\section{Résumé :}

Caulerpa racemosa est une espèce introduite en Méditerranée dont la souche envahissante, C. racemosa var. cylindracea, originaire du sud-ouest australien fut découverte au début des années quatre-vingt-dix en Libye, et s'est largement propagée depuis. A partir de l'été 2005 nous avons constaté la présence de cette espèce dans différentes localités de la région centre de la côte algérienne, et sa répartition sur différents types de substrats et à différentes profondeurs. Depuis un envahissement de plus en plus important peut être observé dans certaines zones. Oculina patagonica est un cnidaire anthozoaire, appartenant à l'ordre des scléractinaires qui serait originaire du sud-ouest de l'atlantique. Cette espèce a été signalée par plusieurs auteurs dans différents sites méditerranéens. L’objectif de ce travail est de décrire les colonies observées dans les baies d'Alger et de Bou Ismail.

\section{Mots-clés :}

Caulerpa racemosa - Oculina patagonica - Espèces introduites - Bou Ismail - Alger

\section{Introduction}

Caulerpa racemosa (figure 1) communément appelée algue raisin est une chlorophycée (algues vertes) de la famille des Caulerpales. Depuis sa découverte au début des années quatre-vingt-dix, en Libye, la souche envahissante de Caulerpa racemosa s'est largement propagée en Méditerranée. Des travaux récents ont démontré que cette variété est Caulerpa racemosa var. cylindracea (Sonder) Verlaque, Huisman et Boudouresque, originaire du sud-ouest australien. Elle a la capacité de se reproduire de façon sexuée ou asexuée par bouturage. Les activités telles que la pêche et l'ancrage des navires constituent les premiers facteurs de sa propagation. Cette espèce colonise plusieurs types de fonds: sable, roche, vase, matte morte de posidonies de 0 à $50 \mathrm{~m}$ de profondeur (CAPIOMONT et al., 2005). 
La connaissance de la Mer :

un vecteur du développement durable en Méditerranée

Oculina patagonica Angelis 1908 (figure 2) est un cnidaire anthozoaire appartenant à l'ordre des Scléractinaires qui n'était connu qu'à l'état fossile du sud-est de l'Argentine (DE ANGELIS D’OSSAT, 1908).

En 1966 l'espèce fut découverte pour la première fois vivante au large des côtes liguriennes à $1 \mathrm{~km}$ du port de Savono (ZIBROWIUS, 1974). Depuis l'espèce a été signalée dans plusieurs localités méditerranéennes. L’importance des colonies espagnoles indique que l'espèce y était présente bien avant sa première signalisation. (ZIBROWIUS \& RAMOS, 1983). Concernant nos côtes, deux colonies ont été découvertes aux îles Habibas. La première a été observée en 2005 et en 2007 (SARTORETTO et al., 2008). Cette espèce aurait réussi son invasion à partir du détroit de Gibraltar vers les eaux levantines par le biais de la navigation.

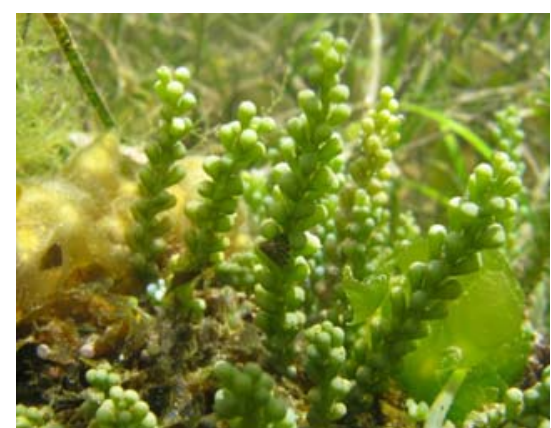

Figure 1. Caulerpa racemosa.

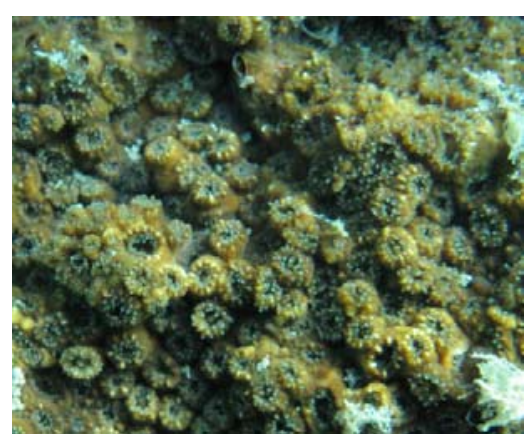

Figure 2. Oculina patagonica.

\section{Matériel et méthodes}

\subsection{Echantillonnage}

Les observations ont été faites, au cours de plusieurs prospections effectuées, à pied, en plongée libre ou en scaphandre dans la région centre de la côte algérienne. Les zones étudiées (figure 3) sont Tamentfoust, Sidi Fredj et Tipaza.

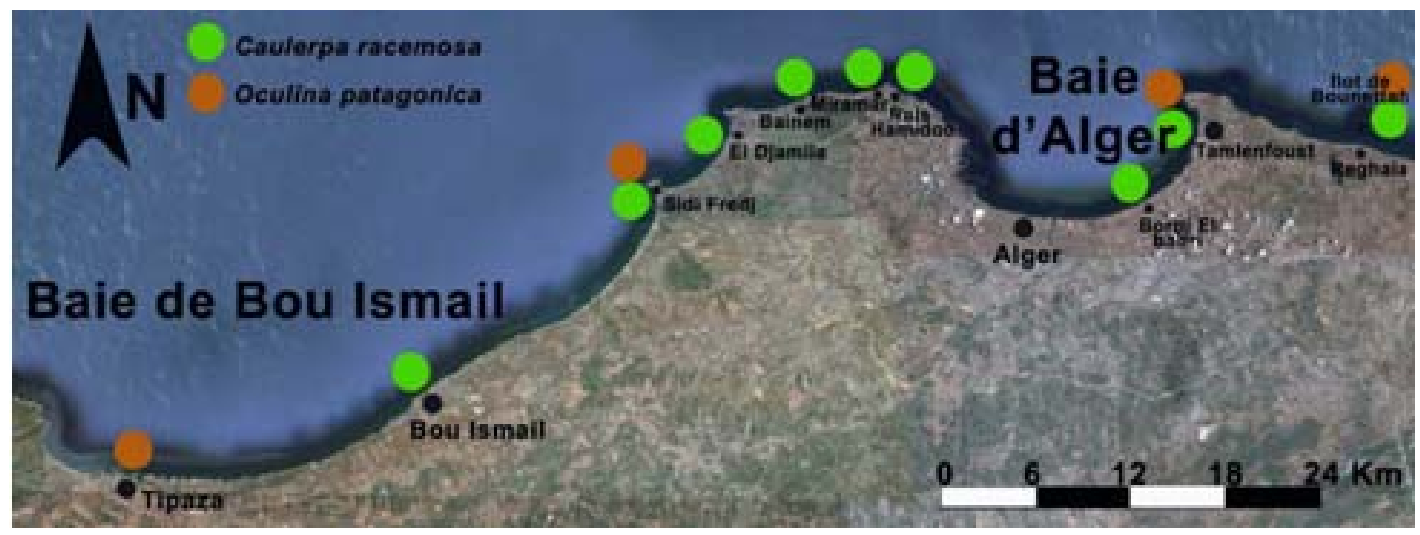

Figure.3. Zones d'échantillonnage. 
Les colonies ont été photographiées, mesurées, et localisées in situ au GPS. Les différents éléments sont intégrés dans un SIG (figure 4)

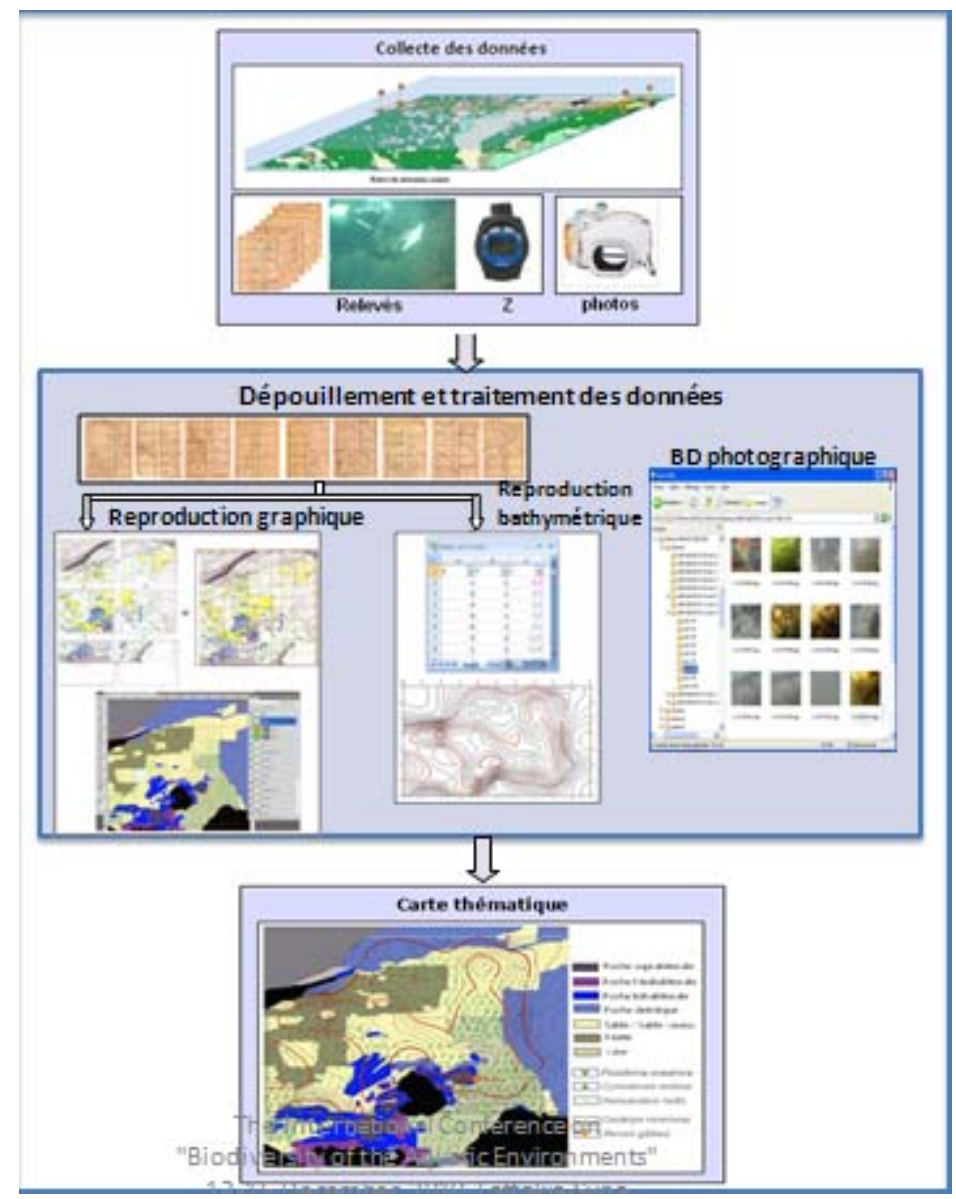

Figure 4. Organigramme du SIG développé.

\subsection{Caulerpa racemosa}

A partir de l'été 2005 nous avons pu observer cette espèce dans différents sites aux environs d’Alger (LAMOUTI, 2006 ; LAMOUTI, 2011). L'espèce a également été signalée par d'autres auteurs (OULD AHMED \& MEINESZ, 2007; SERIDI \& KABRANE, 2010). Une photo prise par des plongeurs et diffusée sur internet indique la présence de l'espèce au large de Décapelage. Concernant le reste de la côte algérienne, l'espèce est présente à Béni-Saf depuis 2008 (détermination à partir de photographies) et récemment à Mostaganem. Les colonies les mieux développées se rencontrent dans les zones abritées. L'espèce a été rencontrée à partir de quelques centimètres de profondeur jusqu'à 29 mètres sur différents types de fonds (figure 3 et tableau 1) : fond dur, fond vaseux, sableux, entre les rhizomes de phanérogames marines, entre les algues médiolittorales inférieures et infralittorales supérieures; sur les mattes mortes et sur divers déchets solides. L'espèce semble également présenter plusieurs aspects 
La connaissance de la Mer :

un vecteur du développement durable en Méditerranée

morphologiques. L'espèce se trouve dans des zones où les activités de pêche et de plaisance sont très développées et contribuent donc très largement à sa propagation. Les comparaisons entre les données obtenues entre 2005 et 2010 indiquent une prolifération importante dans des zones ou l'espèce était peu ou pas représentée (LAMOUTI, 2010). Le tableau résume les résultats des observations en indiquant le lieu, la date d'observation, la profondeur et le type du fond.

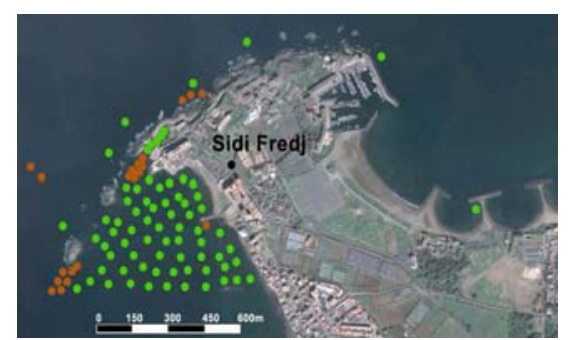

Sidi Fredj

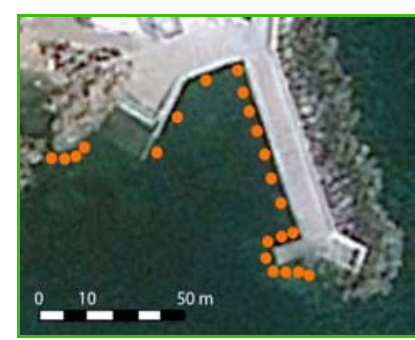

Corne d'or (Tipaza)

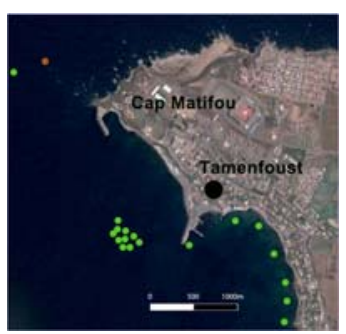

Tamentfoust

Figure 5. Détail de la répartition de Caulerpa racemosa et Oculina patagonica dans la région centre de la côte algérienne à Sidi Fredj, Tipaza et Tamentfoust.

Tableau 1. Présence de Caulerpa racemosa dans la région centre de la côte algérienne.

\begin{tabular}{llll}
\hline Lieu & Date d'observation & Profondeur & Type de fonds \\
\hline Bounettah & $2009-2010$ & $13 m$ & Sable \\
Tamentfoust & $2005,2009-2010$ & $22 m$ & Sablo-vaseux \\
Bordj El Bahri & 2007 & $<1 \mathrm{~m}$ & Roche (Seridi, 2007) \\
RaisHamidou & $2006-2007$ & $1-17 m$ & Roche, herbier, galets \\
Miramar & 2006 & $29 m$ & Roche, herbier, sable \\
Bainem & 2006 & $6 m$ & Roche, herbier \\
El Djamila & 2006 & $8 m$ & Roche, herbier \\
Sidi Fredj & 2005 à 2010 & $1-12 m$ & Vase, sable, herbier, déchets solides \\
Bou Ismail & $2006-2007$ & $<1 m$ & Roche \\
\hline
\end{tabular}

\subsection{Oculina patagonica}

La première observation de l'espèce remonte à août 2008 dans la région de Sidi Fredj mais elle n’a été déterminée que plus tard. Puis d'autres colonies ont été observées (tableau 2 et figure 3), (LAMOUTI, 2011). Les plus grandes colonies se trouvent sur les fonds rocheux naturel et les enrochements, avec des largeurs et longueurs allant de 10 à $50 \mathrm{~cm}$. On les trouvent dans une biocénose à algues infralittorales, occupant un faciès surpâturé à algues encroûtantes et oursins. Certaines colonies présentent des traces de blanchiment. Dans la région de Tipaza (figure 3) l'espèce est très abondante au port de la corne d'or avec plusieurs colonies de taille importante installées sur les parois verticales du port de la Corne d'or. L'expansion de l'espèce est favorisée par sa capacité à s'implanter dans des milieux propres ou pollués, et par son mode de reproduction. 
(SARTORETTO et al., 2008). Des études ultérieures devraient apporter plus d'informations sur sa répartition et les facteurs de sa propagation. Le tableau 2 résume les observations en précisant les dates et les lieux.

Tableau.2. Observations d'Oculina patagonica dans la région centre de la côte algérienne.

\begin{tabular}{|c|c|c|}
\hline Site & Dates d'observation & Coordonnées \\
\hline Corne d'or & Mai 2010 & Lat: $36^{\circ} 35^{\prime} 37^{\prime \prime}$ Long: 2'28'24', \\
\hline Sidi Fredj & $\begin{array}{l}\text { (Aout, Novembre 2008) ;( Mars, } \\
\text { Aout, Septembre 2009) et Octobre } 2010\end{array}$ & Lat: 3645’41', Long: 250’16 ', \\
\hline Tamentfoust & Mai 2010 & Lat: 3648’43’' Long: 3`13’16' \\
\hline Tamentfoust & Décembre 2009 & Lat: 3647’29’’ Long: 3²1'10’’ \\
\hline
\end{tabular}

\section{Conclusion}

Les espèces introduites ne cessent de s'étendre dans les eaux côtières méditerranéennes. Dans ce travail nous avons suivi la répartition spatiotemporelle de deux espèces le long de la côte algéroise. Une base de données géo-référenciée a été créée et mise dans un SIG pour la conserver. Les éléments retenus sont le lieu, les coordonnées géographiques, la date d’observation, la bathymétrie et la nature du fond, précisés par des documents photographiques. En conclusion, la multiplication des prospections devrait apporter plus d'informations sur la répartition de ces espèces qui ont probablement une répartition plus importante. En effet les zones où les prospections ont été effectuées, restent restreintes. Il faudrait développer une méthodologie plus avancée pour pouvoir réaliser une cartographie détaillée de toute la région concernée, à savoir la télédétection à la fois hyperspectrale et à très haute résolution spatiale.

\section{Références bibliographiques}

CAPIOMONT A., BREUGNOT E., DEN HAAN M., MEINESZ A. (2005). Phenology of a deep-water population of Caulerpa racemosa var. cylindracea in the northwestern Mediterranean Sea. Botanica marina, 48, pp 80-83. doi:10.1515/BOT.2005.006 DE ANGELIS D’OSSAT G. (1908). Altri Zoantari del Terziario Della Patagonia. Anales del Museo Nacional de Buenos Aires, 3, pp 93-102.

LAMOUTI S. (2006). Rapport d'activité du réseau de surveillance des herbiers à posidonies, habitats remarquables et espèces invasives. Dir. Grimes, Projet AMIS/SMAP3, APPL/CIRSA.

LAMOUTI S. (2010). Contribution au développement de cartes biocénotiques dans la région centre de la côte algérienne, par la combinaison de méthodes de télédétection et d'observation in situ. Mémoire de magister, USTHB.78 p. 
La connaissance de la Mer :

un vecteur du développement durable en Méditerranée

LAMOUTI S. (2011). Suivi de la limite inférieure de l'herbier à posidonies de l'îlot de Bounettah (Année 2010). Rapport APPL, 31 p.

OULD AHMED N., MEINESZ A. (2007). First record of the invasive alga Caulerpa racemosa (Caulerpales, Chlorophyta) on the coast of Algeria. Cryptogamie, 28 (3), pp 303-305.

SERIDI H. , KABRANE K (2010). Progression de Caulerpa racemosa (Caulerpales, Chlorophyta) sur le littoral algérien, In Actes du 4éme Symposium méditerranéen sur la végétation marine (Yasmine-Hammamet, 2-4 Décembre 2010), PNUE-PAMCAR/ASP, dir. El Asini S, Lamgar H., Belgacem W. édits., CAR/ASP publ.,Tunis, pp 125-128

SARTORETTO S., HARMELIN J.G., BACHET F., BEJAOUI N., LEBRUN O., ZIBROWIUS H. (1974). Oculina patagonica, scléractiniaire hermatypique introduit en Méditerranée. Helgoländer Wissenschaftliche Meeresuntersuchungen 26(2), pp 153-173.

ZIBROWIUS H., RAMOS A. (1983). Oculina patagonica, scléractiniaire exotique en Méditerranée - nouvelles observations dans le Sud-Est de l'Espagne. Rapports Commission Internationale pour l'Exploration Scientifique de la Mer Méditerranée [CIESM] 28(3), pp 297-301. 